\title{
Chaotic transport in Hamiltonian systems perturbed by a weak turbulent wave field
}

\author{
S. S. Abdullaev \\ Institute for Energy and Climate Research-Plasma Physics, Forschungszentrum Jülich GmbH, \\ Association EURATOM-FZJ, Partner in the Trilateral Euregio Cluster, D-52425 Jülich, Germany
}

(Received 27 April 2011; revised manuscript received 13 June 2011; published 15 August 2011)

\begin{abstract}
Chaotic transport in a Hamiltonian system perturbed by a weak turbulent wave field is studied. It is assumed that a turbulent wave field has a wide spectrum containing up to thousands of modes whose phases are fluctuating in time with a finite correlation time. To integrate the Hamiltonian equations a fast symplectic mapping is derived. It has a large time-step equal to one full turn in angle variable. It is found that the chaotic transport across tori caused by the interactions of small-scale resonances have a fractal-like structure with the reduced or zero values of diffusion coefficients near low-order rational tori thereby forming transport barriers there. The density of rational tori is numerically calculated and its properties are investigated. It is shown that the transport barriers are formed in the gaps of the density of rational tori near the low-order rational tori. The dependencies of the depth and width of transport barriers on the wave field spectrum and the correlation time of fluctuating turbulent field (or the Kubo number) are studied. These numerical findings may have importance in understanding the mechanisms of transport barrier formation in fusion plasmas.
\end{abstract}

DOI: 10.1103/PhysRevE.84.026204

PACS number(s): 05.45.Ac, 45.20.Jj, 52.55.Dy, 52.55.Fa

\section{INTRODUCTION}

The study of transport of charged particles in turbulent magnetic and electric fields is of a great interest in laboratory and astrophysical plasmas (see, e.g., [1-7] and references therein). Particularly, in magnetically confined fusion devices one of the great challenges is to reduce the enhanced transport of particles known as anomalous transport which is induced by small-scale turbulent fields generated by instabilities $[1,4]$. Such improved confinement regimes of plasmas has been experimentally found by heating plasma [8,9]. They are achieved due to a creation of radially localized zones with the reduced transport, known as transport barriers (see, e.g., reviews $[2,4,10,11])$.

The important issue of the transport barrier formation in the turbulent plasma is a determination of its dominant mechanisms (see [3] and references therein). At present several possible mechanisms, like an ExB flow shear, a zonal flow, or a magnetic shear, have been proposed. One of these issues is the role of low-order magnetic surfaces in the formation transport barriers which have been discussed in a number of works [12-25].

One of the simplified approaches to the transport problem of charged particles in laboratory and space plasmas are test particle models in which no back reaction of media due to a particle motion is taken into account. These models are widely used to study the particle transport caused by turbulent fields in fusion plasmas (see, e.g., Refs. [26-35] and references therein) and the transport cosmic rays in heliosphere (see Refs. [7,36,37] and references therein). Particularly, in Refs. $[27,28,32,33]$ the test particle models are used to explore the effect of low and reversed magnetic shear on the radial transport of field lines and particles in the presence of magnetic and electrostatic perturbations.

The numerical study of particle motion in a turbulent field encounters practical difficulties related with both the accuracy and computational times. The standard methods to integrate the equations of motion containing a large number of fast-oscillating terms require very long computational times [27]. Therefore, different computational methods have been proposed to overcome these difficulties. Particularly, in Refs. [29-31] a numerical mapping technique [38] has been used to study the relativistic particle's motion in a tokamak plasma perturbed by a turbulence magnetic field. In Refs. $[28,32]$ to study the drift motion of particles in the turbulent electrostatic fields the so-called drift wave maps have been derived. Similar mappings have been used in Refs. $[33,34]$ to explore such a problem. These maps which are similar to the standard map, perturbed twist, and nontwist maps known in Hamiltonian dynamics (see, e.g., [39]) significantly accelerate the calculations.

However, the computational capabilities of these mapping models are limited to include only a moderate number of turbulence wave field modes, at most consisting of a few tens of modes. Therefore, they also encounter difficulties in the studies of the particle's motion in typical electromagnetic turbulence fields of tokamak plasmas which may contain up to thousands of small-scale wave modes.

In the present work we propose a new numerical approach to the transport problem of particles in a small-scale turbulent field which overcomes the above-mentioned difficulties. It is based on the numerical integration of the corresponding equations of motion using a symplectic mapping with a time step equal to one full turn in a phase space of the system. The construction procedure of this mapping is given in Ref. [40].

Specifically, we consider the chaotic transport in an onedegree-of-freedom Hamiltonian system perturbed by a weak turbulent wave field. It corresponds to the simplified problem of the stochastic particle transport in magnetically confined plasmas in the presence of turbulent electromagnetic fields. We assume that the turbulent field contains a large number of modes extending up to several thousands. Moreover, we suppose that the mode phases are fluctuating with a finite correlation time.

The problem itself may also have a theoretical interest as a dynamics of Hamiltonian systems subjected to small 
amplitude time-dependent perturbations with a wide spectrum of frequencies. We will study the role of rational tori on the chaotic transport in such systems. It will be shown that a profile of diffusion coefficients across tori may have a fractal nature. Particularly, the chaotic transport is reduced near the low-order rational tori thereby forming transport barriers. We will study the condition of forming these transport barriers and their dependence on the mode spectra and correlation time of turbulent field.

The paper consists of six sections. The description of a Hamiltonian system, the methods of symplectic integrations, particularly, fast mapping, and the specification of the model of a Hamiltonian system are given in Sec. II. The analysis of transport in a Hamiltonian system caused by the fluctuating turbulence field is described in Sec. III. The density of rational tori and its properties are studied in Sec. IV. The conditions of the transport barrier formation near the loworder rational tori are explored in Sec. V. In the final Sec. VI the summary of obtained results and conclusions are made.

\section{HAMILTONIAN PROBLEM}

In this section we first describe the Hamiltonian system and the assumptions on the turbulence field. Then the two methods of symplectic integration of this Hamiltonian system will be presented.

\section{A. Description of Hamiltonian system}

Consider a one-degree-of-freedom Hamiltonian system which describes a motion in finite domain of the phase space of canonical variables $(q, p)$. This system is integrable with the constant of motion $H_{0}(p, q)=$ const, and one can introduce the action-angle variables $(J, \vartheta)$. The trajectories lie on the closed curve $C_{p}$ in the phase plane $(q, p)$ with $J(q, p)=$ const. The angle variable $\vartheta,(\bmod 2 \pi)$ determining the position of a point on the curve is a linear function of time $\vartheta=\omega(J) t+\vartheta_{0}$, where $\omega(J)=d H_{0}(J) / d J$ is a frequency of motion.

In the presence of time-dependent perturbations the action variable $J$ does not conserve any more; the evolution of variables $(J, \vartheta)$ is governed by the two-dimensional (2D) Hamiltonian system,

$$
\frac{d \vartheta}{d t}=\frac{\partial H}{\partial J}, \quad \frac{d J}{d t}=-\frac{\partial H}{\partial \vartheta}
$$

where $H=H(J, \vartheta, t)$ is the Hamiltonian function of the system. It can be presented as a sum of the unperturbed Hamiltonian $H_{0}(J)$ and the part $H_{1}(J, \vartheta, t)$ corresponding to the perturbations,

$$
H(J, \vartheta, t)=H_{0}(J)+H_{1}(J, \vartheta, t) .
$$

The perturbation Hamiltonian describing the effect of a weak turbulent field can be presented by a Fourier series in $\vartheta$ and $t$,

$$
H_{1}(J, \vartheta, t)=\epsilon \sum_{m, n} H_{m n}(J) \cos (m \vartheta) \cos \left(\Omega_{n} t+\chi_{m n}(t)\right),
$$

where the Fourier coefficients $H_{m n}(J)$ of the $(m, n)$-wave mode with the random phases $\chi_{m n}(t), \Omega_{n}$ is the frequency of the $n$th mode, and $\epsilon$ stands for the dimensionless amplitude of the turbulent field. Furthermore, we will set $\Omega_{n}=n$ which corresponds $2 \pi$-time-periodic perturbations. In magnetic fusion devices $\epsilon$ is less than or of order of $10^{-4}$ to $10^{-3}$.

The turbulent field contains a wide spectrum of modes $(m, n)$ ranging in the interval $m_{\min } \leqslant m \leqslant m_{\max }, n_{\min } \leqslant n \leqslant$ $n_{\max }$ with $m_{\max } \gg 1, n_{\max } \gg 1$. We assume that the $(m, n)$ modes with the different $m$ but the same number $n$ are not independent, but the modes belonging to the different mode $n$ are statistically independent. It allows one to assume that the random phases $\chi_{m n}(t)$ depend only on the mode number $n$ [i.e., $\left.\chi_{n}(t) \equiv \chi_{m n}(t)\right]$. This assumption allows one to present the perturbation Hamiltonian in the form,

$$
\begin{gathered}
H_{1}=\epsilon \sum_{n=n_{\min }}^{n_{\max }} \mathcal{H}_{n}(\vartheta, J) \cos \left(n t+\chi_{n}(t)\right), \\
\mathcal{H}_{n}(\vartheta, J)=\sum_{m=m_{\min }}^{m_{\max }} H_{m n}(J) \cos (m \vartheta) .
\end{gathered}
$$

Furthermore we will assume that the turbulent fluctuations as a stationary random process in time with the correlation time $\tau_{c}$ defined as

$$
\left\langle e^{i\left(\chi_{n}(t+\tau)-\chi_{n^{\prime}}(t)\right)}\right\rangle=\delta_{n, n^{\prime}} e^{-\tau / \tau_{c}}
$$

where $\langle\cdots\rangle$ stands for the averaging over ensemble of realization of phase $\chi$. The turbulent transport of particles depends on the Kubo number $K=\tau_{c} / T$, defined as a ratio of the correlation time $\tau_{c}$ to the unperturbed period of motion $T=2 \pi / \omega(J)$.

One can note that a transport problem of test particles caused by a microscale magnetic turbulence in tokamak plasmas can be reduced to the described Hamiltonian system (1)-(3) (see, e.g., [46]). Then the Hamiltonian equation (1) describes a guiding-center motion in the action-angle variables $(\vartheta, J)$, with the action $J$ normalized to $B_{0} R_{0}^{2}$, where $B_{0}$ is the strength of the toroidal magnetic field at the torus center of radius $R_{0}$. The action $J$ labels also the drift surfaces. The angle $\vartheta$ and the time $t$ would correspond to the poloidal and the toroidal angles of the torus, respectively. The frequency $\omega(J)$ would be a winding number, that is, a ratio of the poloidal turns of a guiding-center orbit per one toroidal turn on the given drift surface $J$.

\section{B. Symplectic integration}

The Hamiltonian system given by Eqs. (1)-(3) can be integrated using the symplectic mapping described in Refs. [40-42]. Let $\left(\vartheta_{k}, J_{k}\right)$ be the values of canonical variables $(\vartheta, J)$ at the time instants $t_{k}=k \Delta t,(k=0, \pm 1, \pm 2, \ldots)$, where $\Delta t$ is a mapping step. The evolution of the system is described by the mapping,

$$
\left(\vartheta_{k}, J_{k}\right) \rightarrow\left(\vartheta_{k+1}, J_{k+1}\right)
$$

The general symplectic form of the mapping given by Eq. (6) obtained by the method of canonical transformation 
of variables has the following form:

$$
\begin{gathered}
\psi_{k}=J_{k}-\frac{\partial S_{k}}{\partial \vartheta_{k}}, \quad \Theta_{k}=\vartheta_{k}+\frac{\partial S_{k}}{\partial \psi_{k}}, \\
\bar{\Theta}_{k}=\Theta_{k}+\left(t_{k+1}-t_{k}\right) \omega\left(\psi_{k}\right), \\
J_{k+1}=\psi_{k}+\frac{\partial S_{k+1}}{\partial \vartheta_{k+1}}, \quad \vartheta_{k+1}=\bar{\Theta}_{k}-\frac{\partial S_{k+1}}{\partial J_{k+1}},
\end{gathered}
$$

where $S_{k} \equiv S_{k}\left(\vartheta_{k}, \psi_{k}\right), S_{k+1} \equiv S_{k}\left(\vartheta_{k+1}, \psi_{k}\right)$ are values of the generating function $S=S(\vartheta, \psi, t, \epsilon)$ at $t=t_{k}$ and $t=t_{k+1}$, respectively. The generating function $S$ of this mapping is found as a solution of the corresponding Hamilton-Jacobi equation in a finite interval $t_{k} \leqslant t \leqslant t_{k+1}$. The generating function in the first order of the perturbation parameter $\epsilon$ is given in Refs. [40-42]. However, this generating function is not convenient for the practical calculations for the Hamiltonian systems containing a large number of modes like in Eq. (3). In this case it is convenient to use the formula for the generating function obtained in Ref. [43]. It has the following form:

$$
\begin{gathered}
S_{k}=S\left(\vartheta_{k}, J_{k}, t\right), \\
S_{k+1}=-S\left(\vartheta_{k+1}, \psi_{k}, t_{k+1}\right), \\
S(\vartheta, \psi, t)=\frac{\Delta t}{2} H_{1}(\vartheta, \psi, t) .
\end{gathered}
$$

Numerical simulations of particle transport in a turbulent field with a large number of $(m, n)$ modes encounter mainly two kinds of difficulties related to the accuracy and computational times. In the problems of particle transport in a turbulent field the number $n$ can be of order of $10^{2}$ to $10^{3}$. It would require one to take a very small step $\Delta t$ in the direct numerical integration of the equations of motion with sufficient accuracy, and therefore, enormous computational times.

\section{Fast symplectic mapping}

To avoid difficulties we employ a fast symplectic mapping to integrate the Hamiltonian system (1) (see Refs. [40,44,45]). The mapping is constructed via canonical transformation of variables and has a large integration step equal to one full turn in the angle $\vartheta$.

Let $P_{k}$ be the $k$ th crossing point of an orbit with the section $\vartheta=0$ as schematically shown in Fig. 1. The mapping $P_{k} \rightarrow P_{k+1}$ relates the point $P_{k}$ with the next crossing point $P_{k+1}$. Suppose that $\left(h_{k}, t_{k}\right)$ are the values of the Hamiltonian $h=H_{0}(J)$ and time $t$ at the crossing point $P_{k}$ of the orbit,

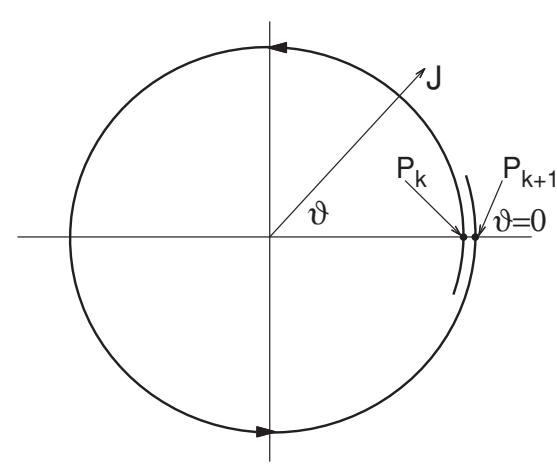

FIG. 1. Schematic view of the mapping, $P_{k} \rightarrow P_{k+1}$. respectively. Then for the small perturbation parameter $\epsilon \ll 1$ the simplified form of the mapping $P_{k} \rightarrow P_{k+1}$ reads

$$
\begin{aligned}
h_{k+1}= & h_{k}-\epsilon \frac{\partial}{\partial t_{k}} P\left(h_{k+1}, t_{k}+\pi / \omega\left(h_{k}\right)\right), \\
t_{k+1}= & t_{k}+\pi\left[1 / \omega\left(h_{k}\right)+1 / \omega\left(h_{k+1}\right)\right] \\
& +\epsilon \frac{\partial}{\partial h_{k+1}} P\left(h_{k+1}, t_{k}+\pi / \omega\left(h_{k}\right)\right),
\end{aligned}
$$

where the generalized Poincare integral $P(h, t)$ is taken along the closed contour of the unperturbed orbit. It can be presented in the form,

$$
\begin{aligned}
P(h, t) & =\int_{0}^{2 \pi / \omega} H_{1}\left(h, t+t^{\prime}\right) d t^{\prime} \\
& =\sum_{n=n_{\min }}^{n_{\max }} R_{n}(h) \exp \left(i n t+i \chi_{n}(t)\right) .
\end{aligned}
$$

Using the expression for the perturbation Hamiltonian (3) the coefficients $R_{n}(h)$ can be reduced to

$$
R_{n}(h)=\frac{\pi}{\omega(h)} \sum_{s= \pm 1} \sum_{m=m_{\min }}^{m_{\max }} H_{m n}(J) \frac{e^{i x_{m n}^{(s)}}-1}{i x_{m n}^{(s)}},
$$

where $x_{m n}^{(s)}=(m-s n / \omega) 2 \pi$. Extending the sum in (11) from $m_{\text {min }}=1$ to $m_{\max }=\infty$ the coefficients $R_{n}(h)$ can be presented as a sum of regular $R_{n}^{(\mathrm{reg})}$ and oscillatory $R_{n}^{(\mathrm{osc})}$ parts:

$$
\begin{gathered}
R_{n}=R_{n}^{(\mathrm{reg})}+R_{n}^{(\mathrm{osc})}, \\
R_{n}^{(\mathrm{reg})}=(\pi / \omega) H_{n}(n / \omega), \\
R_{n}^{(\mathrm{osc})}=\left(\cos ^{2}\left(\frac{\pi n}{\omega}\right)-1+i \frac{\sin (2 \pi n / \omega)}{2}\right) R_{n}^{(\mathrm{reg})},
\end{gathered}
$$

where $H_{n}(m) \equiv H_{m n}(J)$ is a function defined by extending the value of integer number $m$ to the continuous ones. One should note that at the resonant tori $J_{m n}, m \omega\left(J_{m n}\right)=n$, the oscillatory part $R_{n}^{(\text {osc })}=0$.

The finite correlation time $\tau_{c}$ of the phases $\chi_{n}$ are taken into account by randomly changing them after the $M$ mapping steps equal to the integer part of the Kubo number $K$ (i.e., $M=[K])$. However, the mapping cannot consider the Kubo number less than one.

\section{Model}

For the numerical study of the transport we should specify models for the unperturbed Hamiltonian system and the turbulent field. Below we consider the models corresponding to the particle dynamics in magnetically confined plasmas in the presence of weak magnetic perturbations (see, e.g., Ref. [46]).

The unperturbed Hamiltonian is characterized by the frequency of motion $\omega(J)$. Furthermore, we will choose the action variable $J$ normalized to its maximal possible value, so that it changes in the interval $0<J<1$. We consider the two cases of the dependence of the inverse frequency $\omega(J)$ on $J$ :

(i) the monotonic linear dependence $1 / \omega(J)=q_{0}+$ $\left(q_{a}-q_{0}\right) \mathrm{J}$

(ii) the nonmonotonic dependence $1 / \omega(J)=q_{m}+$ $\beta\left(J-J_{m}\right)^{2}$ with the minimal value at off-axis value $J_{m}$.

Here, $q_{0}, q_{a}, q_{m}$, and $\beta$ are constant parameters. 
The amplitude of turbulent field mode is chosen as a localized in $J$ function in the form,

$$
H_{m n}(J)=\frac{c_{n}}{m} \exp \left[-\frac{(m \gamma(J)-n / \omega(J))^{2}}{4 w_{n}^{2}}\right],
$$

where $w_{n}$ describes the width of the mode, $c_{n}$ is the amplitude of toroidal modes which we will choose in the power-law form $c_{n}=n^{-\alpha}$ with a constant $\alpha$. The function $\gamma(J)$ describes a deviation of the location $J_{\max }$ of the maximal value $c_{n} / m$ of the amplitude $H_{m n}(J)$ from the corresponding resonant tori $J_{m n}, \omega\left(J_{m n}\right)=n / m$. If $\gamma(J) \equiv 1$ the amplitude reaches it maximum at the resonant tori. We choose the function $\gamma(J)$ as follows:

$$
\gamma(J)=\gamma_{0}\left(1+\kappa\left(J-J_{c}\right)^{2}\right),
$$

where $\gamma_{0}, 0<J_{c}<1, \kappa>0$ are constant parameters.

According to Eq. (12) the function $R_{n}^{(\mathrm{reg})}(h)$ for the model (13) is reduced to

$$
R_{n}(h)=\frac{c_{n} \omega(J)}{n} \exp \left[-\frac{n^{2}(\gamma(J)-1)^{2}}{4 w_{n}^{2} \omega^{2}(J)}\right] .
$$

From (15) follows that for $\gamma(J) \equiv 1$ the function $R_{n}^{(\mathrm{reg})}(h)$, and therefore the Poincaré integral $P(h, t)$ does not depend on the width $w_{n}$ of the perturbation amplitude.

The model (13) for the amplitude $H_{m n}(J)$ allows one to obtain the closed analytical formula for the perturbation functions $\mathcal{H}_{n}(\vartheta, J)$ in the perturbation Hamiltonian (4). Using the Poisson summation rule the sum over $m$ in Eq. (4) can be reduced to

$$
\begin{aligned}
\mathcal{H}_{n}(\vartheta, J)= & c_{n} \sum_{p=-\infty}^{\infty} \int_{m_{\min }}^{m_{\max }} m^{-1} \exp \left[-\frac{(m \gamma-n / \omega(J))^{2}}{4 w_{n}^{2}}\right] \\
& \times \cos (m \vartheta) \cos (2 \pi m p) d m .
\end{aligned}
$$

Replacing $m$ by $m=n \omega(J) / \gamma(J)$ in the pre-exponent of the integral in Eq. (16), and taking $m_{\min }=0$ and $m_{\max } \rightarrow \infty$, the function $\mathcal{H}_{n}(\vartheta, J)$ can be approximated by

$$
\mathcal{H}_{n}(\vartheta, J) \approx 2 \sqrt{a \pi} c_{n} \sum_{p=-\infty}^{\infty} f(a, b, \vartheta-2 \pi p),
$$

where

$$
\begin{gathered}
f(a, b, \vartheta)=e^{-a \vartheta^{2}} \cos (b \vartheta), \\
a=w_{n}^{2} / \gamma^{2}, \quad b=n /(\gamma \omega(J)) .
\end{gathered}
$$

This analytical formula would accelerate a numerical calculation of the symplectic integrator (7). In practical calculations it is sufficient to take only a few terms $p=0, \pm 1$ in the sum over $p(17)$. However, one should note that such a presentation of $\mathcal{H}_{n}(\vartheta, J)$ is valid only for the large number modes $n \gtrsim 10$.

\section{E. Comparison of mapping with small-step integration}

For the model described above we compared the results of calculations of the second moment of displacements of the action $J$,

$$
\sigma_{0}^{2}(t)=\left\langle(J(t)-J(0))^{2}\right\rangle=\frac{1}{N_{t}} \sum_{i=1}^{N_{t}}\left(J_{i}(t)-J(0)\right)^{2},
$$

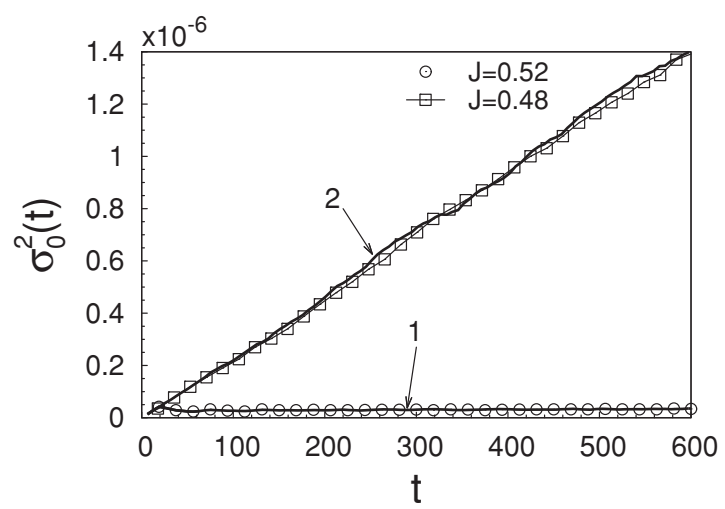

FIG. 2. Dependencies of $\sigma_{0}^{2}(t)$ on $t$ for the model (13) obtained using the symplectic integrator (7) with the time step $\Delta t=2 \pi / 128$ (solid curves 1 and 2) and the fast mapping (9) ( circles and squares). Solid curve 1 and circles correspond to the tori $J_{0}=0.52$, and solid curve 2 and squares correspond to the tori $J_{0}=0.48$. The parameters of the model are $q_{0}=0.8, q_{a}=5,20 \leqslant n \leqslant 50, \gamma(J) \equiv 1$, and $\epsilon=10^{-4}$.

obtained by the fast mapping (9) and the symplectic integrator $(7)$. Here $\langle(\cdots)\rangle$ stands for averaging over the initial phases $\chi_{n}(0), n_{\min } \leqslant n \leqslant n_{\max }$, randomly chosen in the interval $[0,2 \pi], J_{i}(t)$ is the orbit corresponding to the $i$ th trial, and $N_{t}$ is a total number of trials.

The calculations are carried out for the radial profile $(i)$ of $1 / \omega(J)$, and the values of parameters are chosen $q_{0}=0.8$, $q_{a}=5$, in Eq. (13) the function $\gamma(J) \equiv 1$ and $w_{n}=\infty$, the exponent $\alpha=5 / 6,20 \leqslant n \leqslant 50$, and the turbulence level $\epsilon=$ $10^{-4}$. For the given parameters the relative accuracy $10^{-4}$ of $\sigma_{0}^{2}(t)$ calculated by the symplectic integrator (7) is reached with the time step $\Delta t=2 \pi / 128$.

Figure 2 shows the comparison $\sigma_{0}^{2}(t)$ calculated by the fast mapping (9) (circles and squares) and the symplectic integrator (7) (solid curves 1 and 2) with the time step $\Delta t=2 \pi / 128$ at the two positions of tori $J_{0}$ : solid curve 1 and circles correspond $J_{0} \equiv J(0)=0.52$, solid curve 2 and open squares correspond to $J_{0}=0.48$. As seen from Fig. 2 the fast mapping quite accurately reproduces the results of the small time-step symplectic integration for the two different types of motion corresponding to the diffusive and regular regimes (see Secs. III and V).

However, the small time-step symplectic integration takes almost three or four orders longer computation time than the fast mapping. It would require several weeks of calculations in the standard PCs to obtain a radial dependence of diffusion coefficients for the model with the modest number of modes. It becomes practically impossible to use the symplectic integration in the case with a large number of modes extending up to several thousands. Furthermore, we will use the fast mapping to study our problem.

\section{ANALYSIS OF CHAOTIC AND FLUCTUATING TRANSPORT REGIMES}

The transport of across tori, that is, along the $J$ axis, is caused by the following: (i) the chaotic motion due to the interactions of neighboring islands created by the nonlinear resonance of particle motion with the corresponding $(m, n)$ 
mode of the turbulent field; and (ii) the turbulent fluctuations due to a finite correlation time $\tau_{c}$. In this section we analyze the interplay between two of the transport mechanisms.

\section{A. Quasilinear approximation for chaotic transport}

At the large Kubo numbers $K \gg 1$ when the correlation time $\tau_{c}$ is much longer than the oscillation period $T=2 \pi / \omega$ (i.e., $\tau_{c} \gg T$ ), the chaotic motion mainly contributes to the radial transport. The latter starts from the certain value of the turbulence level $\epsilon$ which depends on the spectrum of the turbulent field. The critical level of the turbulence $\epsilon_{c}$ can be qualitatively described by the overlapping (Chirikov) criteria, $\sigma_{\text {Chir }} \approx 1$, where $\sigma_{\text {Chir }}$ is the parameter characterizing the degree of overlapping of neighboring islands, that is,

$$
\begin{aligned}
\sigma_{\text {Chir }} & =\frac{\Delta J_{m n}+\Delta J_{m^{\prime} n^{\prime}}}{2\left|J_{m n}-J_{m^{\prime} n^{\prime}}\right|}, \\
\Delta J_{m n} & =4\left|\frac{\epsilon H_{m n}\left(J_{m n}\right)}{\omega^{\prime}\left(J_{m n}\right)}\right|^{1 / 2},
\end{aligned}
$$

where $\Delta J_{m n}$ is the width of the $(m, n)$ island, $J_{m n}$ are the resonance values of the action $J, \omega\left(J_{m n}\right)=n / m$, and $(m, n)$ and $\left(m^{\prime}, n^{\prime}\right)$ are neighboring islands, $\omega^{\prime}(J) \equiv d \omega / d J$.

The transport along $J$ can be characterized by the diffusion coefficient $D(J)=\sigma_{0}^{2}(J) / 2 t$. For the large Kubo numbers $K \gg 1$ and $\sigma_{\text {Chir }} \gg 1$, the diffusion coefficient can be described by the following quasilinear formula:

$$
\begin{aligned}
D(J) & =\frac{\pi \epsilon^{2}}{2 \omega} \sum_{m, n} m^{2}\left|H_{m n}(J)\right|^{2} \delta(m \omega-n) \\
& =\frac{\epsilon^{2}}{8 \pi \omega} \sum_{n} n^{2}\left|R_{n}^{(\mathrm{reg})}(J)\right|^{2}
\end{aligned}
$$

where the second line is obtained using the relations in Eq. (12).

The numerical calculations of diffusion coefficients $D(J)$ are carried out by fitting the calculated second moment of radial displacements $\sigma_{0}^{2}(t)$ as a function of $t$ with the linear function $2 D(J) t$ at the times $t>t_{K}$, where $t_{K}$ is the decorrelation time of phases $\phi_{n}=n t$. The phase decorrelation time $t_{K}$ is equal to the e-folding time of the phase correlation function $C_{n}(t)=\left\langle e^{i\left[\phi_{n}(t)-\phi_{n}(0)\right]}\right\rangle$. The procedures of the determination of the diffusion coefficients and $t_{K}$ are shown in Fig. 3.

Figures 4(a) and 4(b) show the profiles of the Chirikov parameter $\sigma_{\text {Chir }}$ and diffusion coefficients $D(J)$ along the action $J$ at the turbulence level $\epsilon=10^{-3}$, respectively. The diffusion coefficients are calculated using the mapping (9) with the two choices of the Poincare integrals: curve 2 (red) corresponds to the case with $R_{n}=R_{n}^{(\text {reg })}$, that is, when the oscillatory part, $R_{n}^{(\mathrm{osc})}$, is neglected, curve 3 (blue) corresponds to the case when $R_{n}^{(\mathrm{osc})}$ is included (i.e., $R_{n}=R_{n}^{(\mathrm{reg})}+R_{n}^{\text {(osc) }}$ ). The quasilinear values are plotted by curve 1 (solid line). The right-hand axis shows the profile of $1 / \omega(J)$.

As seen from Fig. 4(b) the radial profiles of $D(J)$ along $J$ are described by the fractal-like, irregularly curves which closely follow the quasilinear formula (21) in the regions of $J$ where the Chirikov parameter takes large values $\sigma_{\text {Chir }}>1$. However, in the narrow regions located near the

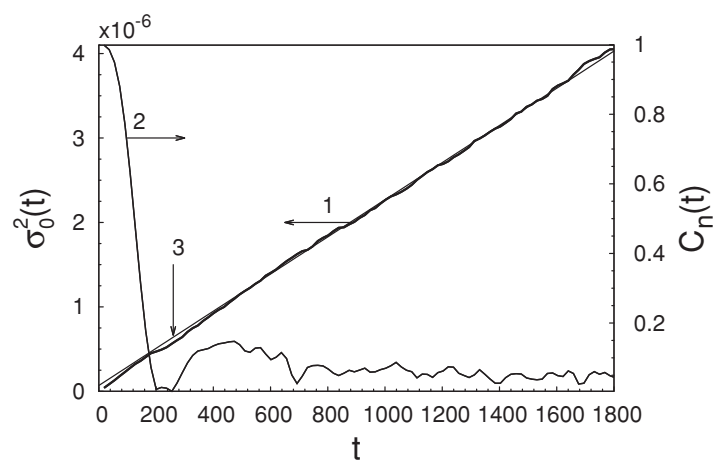

FIG. 3. Determination of the diffusion coefficient $D(J)$. Curve 1 (thick solid line) corresponds to the calculated $\sigma_{0}^{2}(t)$ (left-hand axis); curve 2 corresponds the correlation function of phases $C_{n}(t)$ for $n=$ 20 (right-hand axis); curve 3 (thin solid line) corresponds to the linear function $2 D(J) t$ obtained by fitting of $\sigma_{0}^{2}(t)$ in the interval $600 \leqslant t \leqslant$ 1800. The other parameters are the same as in Fig. 2 and $J_{0}=0.5$.

low-order rational tori $J_{m n}, 1 / \omega\left(J_{m n}\right)=m / n$ with the values $1,3 / 2,2,5 / 2$ the diffusion coefficients become very small or almost negligible, thereby forming the transport barriers. The Chirikov parameter also drops near these rational tori (see
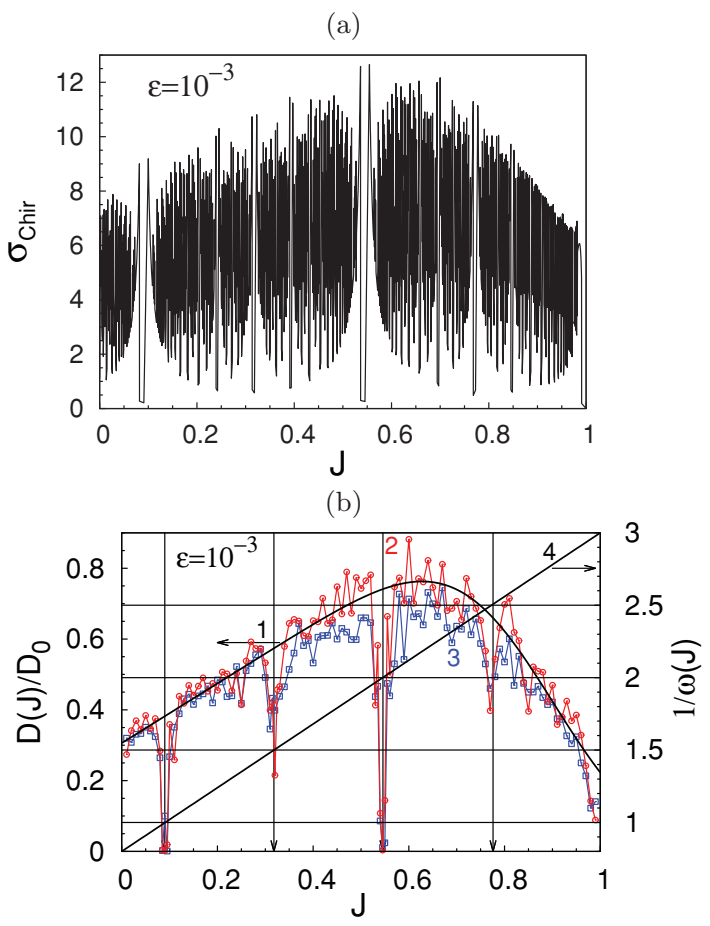

FIG. 4. (Color online) (a) Profile of the Chirikov parameter $\sigma_{\text {Chir }}$ along the action variable $J$. (b) Radial profiles of diffusion coefficients, $D(J)$ (left-hand axis) for the model (13), (14). The values of the model parameters are $q_{0}=0.8, q_{a}=3, \gamma_{0}=1, \kappa=0.2$, $J_{c}=0.2, \alpha=5 / 6, w_{n}=6, \epsilon=10^{-3}$, the modes $20 \leqslant n \leqslant 50$, and the Kubo number $K=100$. The number of trials $N_{t}=2 \times 10^{3}$. Curve 1 (black solid) describes the quasilinear approximation (21); curves 2 [red (circle) ] and 3 (blue open square) correspond to the fast mapping (9) with $R_{n}=R_{n}^{(\mathrm{reg})}$ and $R_{n}=R_{n}^{\text {(reg) }}+R_{n}^{\text {(osc) }}$, respectively; curve 4 describes the profile of $1 / \omega(J)$ (right-hand axis). The vertical thin lines show the locations of main rational resonances, $J_{m n}$, $\left[1 / \omega\left(J_{m n}\right)=m / n=1,3 / 2,2,5 / 2\right]$. 
Fig. 4). We will analyze the nature of these transport barriers in the next sections.

One should note that the approximations of the Poincare integrals $R_{n}$ by $R_{n}=R_{n}^{(\mathrm{reg})}$ and $R_{n}=R_{n}^{(\mathrm{reg})}+R_{n}^{(\mathrm{osc})}$, given by curves 2 and 3 , respectively, give qualitatively close results for the diffusion coefficients. It is related to the fact that for the large Kubo numbers $K \gg 1$ the quasilinear diffusion coefficients (21) are determined only by the regular part of the Poincaré integral $R_{n}^{(\mathrm{reg})}$. Therefore, in these cases one can neglect the oscillatory part $R_{n}^{(\mathrm{osc})}$ of $R_{n}$.

\section{B. Transition from the fluctuating to the chaotic regime}

For the small Kubo number $K$ the transport is mainly caused by the fluctuating in the time turbulent field. For the Kubo number $K=1$ the diffusion coefficients can be obtained from the mapping (9). Using the expansion (10) and the first equation in Eq. (9) the change of the variable $h$ in one mapping turn $\Delta t=2 \pi / \omega(J)$ can be presented as

$$
\begin{gathered}
\Delta h_{k}=h_{k+1}-h_{k}=\epsilon \sum_{n} n\left|R_{n}\right| \cos \Phi_{n}, \\
\Phi_{n}=n t_{k}+\pi / \omega\left(h_{k}\right)+\chi_{n},
\end{gathered}
$$

where $\chi_{n}$ are random phases uniformly distributed in the interval $[0,2 \pi]$. The averaged values of $\Delta h_{k}$ over phases $\chi_{n}$ are zero, $\left\langle\Delta h_{k}\right\rangle=0$. The second moment of $\Delta h_{k}$ is equal to

$$
\sigma_{h}^{2}=\left\langle\left(\Delta h_{k}\right)^{2}\right\rangle=\frac{\epsilon^{2}}{2} \sum_{n} n^{2}\left|R_{n}\right|^{2} .
$$

Using the relations between the variables $h$ and $J$, that is, $\Delta h=(d h / d J) \Delta J=\omega \Delta J$, and the corresponding diffusion coefficients $D(J)$ and $D(h)=\sigma_{h}^{2} / 2 \Delta t$, we arrive at

$$
D(J)=\frac{\epsilon^{2}}{8 \pi \omega} \sum_{n} n^{2}\left|R_{n}\right|^{2} .
$$

This formula for $D(J)$ similar to Eq. (21) in which the integral $R_{n}^{(\text {reg })}$ is replaced by $R_{n}$. The numerical calculation $D(J)$ for the Kubo number $K=1$ is indeed agreed with the formula (24).

One expects that by increasing the Kubo number $K$ the chaotic transport caused by the interaction of resonances starts to contribute to the transport if the turbulence level $\epsilon$ exceeds the critical level $\epsilon_{c}$ qualitatively determined by the criteria $\sigma_{\text {Chir }}\left(\epsilon_{c}\right) \approx 1$. In order to explore this we consider the dependence of the diffusion coefficient on the Kubo number for the three different values of the turbulence level $\epsilon$ : (1) $\epsilon=10^{-5}$; (2) $\epsilon=10^{-4}$; (3) $\epsilon=10^{-3}$ at which the Chirikov parameter $\sigma_{\text {Chir }}$ is (1) less than unity $\sigma_{\text {Chir }} \lesssim 1$; (2) slightly greater than $1 \sigma_{\text {Chir }} \gtrsim 1$; and (2) much larger than $1 \sigma_{\text {Chir }} \gg 1$. Figure 5 shows the dependencies of the diffusion coefficients $D(J)$ on the Kubo number at the fixed tori $J_{0}=0.2$ for these values of $\epsilon$ : Curve 1 corresponds to $\epsilon=10^{-5}$, curve 2 to $\epsilon=10^{-4}$, curve 3 to $\epsilon=10^{-3}$, and curve 4 (a straight dotted line) corresponds to the quasilinear estimation of $D(J)(21)$. Since the diffusion coefficient $D$ is proportional to $\epsilon^{2}$ for the convenience of comparison it is normalized to $\epsilon^{2}$.

For the small level of turbulence which does not produce the chaotic transport, as in the case $\epsilon=10^{-5}$ with $\sigma_{\text {Chir }} \approx 0.55$ the

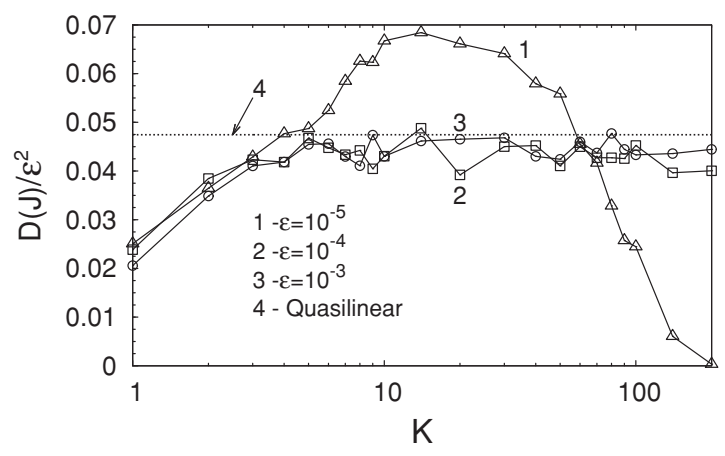

FIG. 5. Dependence of the diffusion coefficients $D(J)$ normalized to $\epsilon^{2}$ on the Kubo number $K$ at the given value $J=0.2$ for three different values of the turbulence level $\epsilon$. Curve 1 (up-triangle) corresponds to $\epsilon=10^{-5}$, curve 2 (square) corresponds to $\epsilon=10^{-4}$, curve 3 (circle) corresponds to $\epsilon=10^{-3}$, and curve 4 (a straight dotted line) corresponds to the quasilinear estimation of $D(J)$ for the large Kubo number $K \gg 1$.

diffusion coefficient $D(J)$ grows with $K$ as $D(J) \sim K \sim \tau_{c}$ in the interval $1 \leqslant K \lesssim 5$, and it reaches the maximum at $K \approx 10$ to 30 then it decays for $K>40$. For the moderate and large turbulence levels, $\epsilon=10^{-4},\left(\sigma_{\mathrm{Chir}} \approx 1.33\right)$ and $\epsilon=$ $10^{-3},\left(\sigma_{\text {Chir }} \approx 5.5\right)$, which may lead to the chaotic transport, the diffusion coefficient reaches its "stationary" level given by the quasilinear value $(21)$ at the the Kubo numbers $K$ exceeding 5 to 6 .

\section{DENSITY OF RATIONAL TORI}

As we have shown above at the large Kubo numbers $K \gg 1$ the transport of particles across tori is mainly caused due to their interactions of nonlinear resonances formed at the resonant tori $J=J_{m n}$. The strength of the interaction depends not only on the level of turbulence $\epsilon$, but also on the density of rational tori. In other words, the chaotic transport rate, a diffusion coefficient, depends on how the resonant tori $J=J_{m n}$ are dense along the axis $J$. Below we calculate the density of rational tori and study its property.

Let $J_{m n}$ be resonant values of tori, $q=1 / \omega\left(J_{m n}\right)=m / n$, corresponding to the mode numbers $(m, n)$. Assume that the interval of the $n$ modes are $M \equiv n_{\text {min }} \leqslant n \leqslant N \equiv n_{\text {max }}$. Let $\Delta W$ be a number of rational tori $J_{m n}$ in the interval $J, J+$ $\Delta J$. Then the density $\rho(J, M, N)$ of rational tori is defined as $\rho(J, M, N)=\Delta W / \Delta J$. One can also introduce the density of rational numbers $q=m / n$, with $n$ in the interval $M \leqslant n \leqslant$ $N$, and $m$ that are coprime to $n$, that is, having no common positive factors other than 1 . It is defined as $\rho(q, M, N)=$ $\Delta W / \Delta J$. The relation between $\rho(J, M, N)$ and $\rho(q, M, N)$ is given by

$$
\rho(J, M, N)=\rho(q, M, N) \frac{d q}{d J} .
$$

As seen from Eq. (25) that the density of rational tori $\rho(J, M, N)$ along the action variable $J$ depends on the shear $d q / d J=-\left(1 / \omega^{2}\right) d \omega / d J$ of the inverse frequency of motion $\omega(J)$ as well as on the density of rational numbers $q=$ $m / n$ with $M \leqslant n \leqslant N$. The former is prescribed by the unperturbed system $H_{0}(J)$ while the latter is determined by the 
(a)

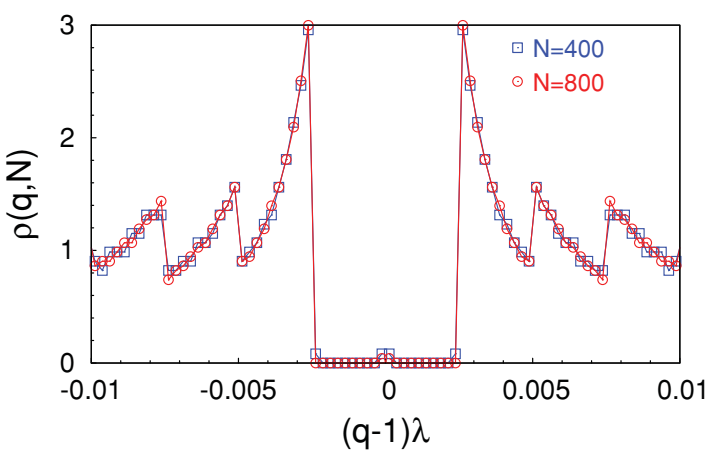

(b)

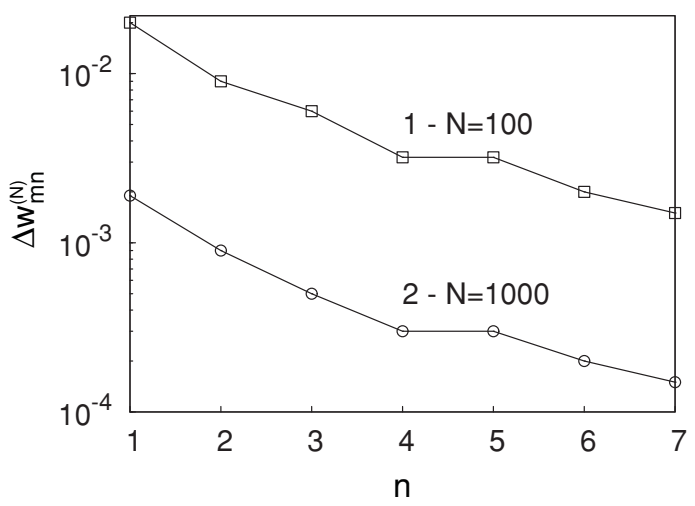

FIG. 6. (Color online) (a) Density distribution function $\rho(q, N)$ of rational numbers $q=m / n(1 \leqslant n \leqslant N)$ for the two different numbers $N$ near the $q=1$. The blue (square) corresponds to $N=400$ and the red (circle) corresponds to $N=800$. The rescaling parameter $\lambda=N / 400$. (b) Gap widths $w_{m n}^{(N)}$ in $\rho(q, N)$ near the rational numbers $1 / n, n=1,2, \ldots, 7$. Curve 1 corresponds to $N=100$; curve $2-$ $N=1000$.

minimal $n_{\min }$ and maximal $n_{\max }$ mode numbers of a turbulence field.

In order to study the property of the density function $\rho(q, M, N)$ it is sufficient to calculate it for the rational numbers in the interval $0 \leqslant q \leqslant 1$. We have numerically calculated the density function $\rho(q, M=1, N)$ for the different values $N$. The numerical calculations show that there are gaps in the density distribution function $\rho(q, M, N)$ near the low-order rational numbers $m / n: 1 / 1,1 / 2,1 / 3$, and so on. As an example the gap near $q=1$ is shown in Fig. 6(a): The square (blue) corresponds to $N=400$ and the circle (red) corresponds to $N=800$. The rescaling parameter $\lambda=N / 400$. The widths of gaps $\Delta w_{m n}^{(N)}$ near rational numbers $q=m / n$ decrease with increasing $n$ as shown in Fig. 6(b) for the two different numbers $N$ : curve 1 corresponds to $N=100$ and curve 2 corresponds to $N=1000$.

Moreover, it was found that the function $\rho(q, M, N)$ has a self-similar behavior near the low-order rational numbers $q_{m n}=1,1 / 2,1 / 3$, and so on. The self-similarity of $\rho(q, N) \equiv$ $\rho(q, M=1, N)$ is manifested by the rescaling law,

$$
\rho\left(q-q_{m, n}, N\right) \approx \rho\left(\frac{q-q_{m, n}}{\lambda}, \lambda N\right)
$$

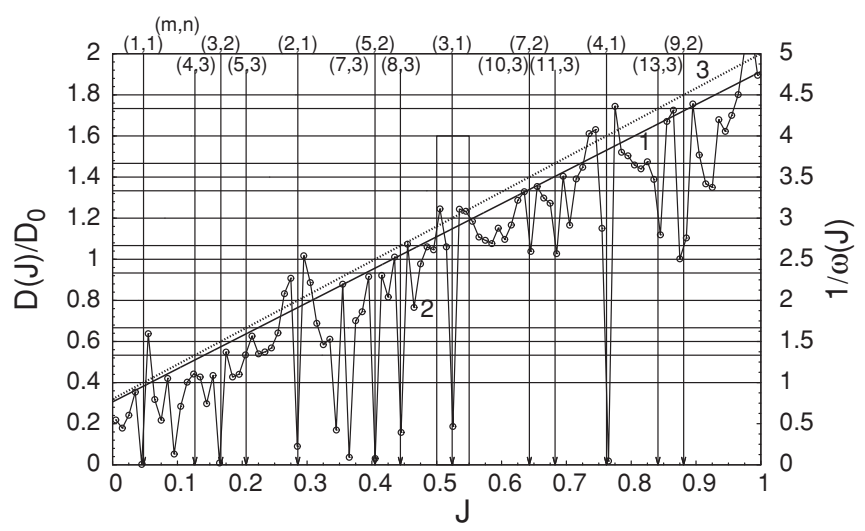

FIG. 7. (Color online) Radial profile of diffusion coefficient for the Kubo number $K=100$. Curve 1 corresponds to the quasilinear values, curve 2 displays the numerical results, curve 3 corresponds to the period of motion $T(J)$ (right-hand axis). The positions of the loworder rational tori $J_{m n},\left(\omega\left(J_{m n}\right)=n / m\right)$, are shown by vertical straight lines. The averaging is done over $2 \times 10^{3}$ orbits. The normalization constant $D_{0}=10^{-9}$.

where $\lambda$ is a constant. The rescaling law (26) for the density distribution function $\rho(q, N)$ near the low-order rational number $q=1$ is shown in Fig. 6(a) for the two values of $N: N=400$ [blue (square)] and $N=800$ [red (circle)]. The rescaling parameter in this case is $\lambda=N / 400{ }^{1}$

As we will show in the next section for the large Kubo numbers $K \gg 1$ the gaps in the density of rational tori strongly affect the chaotic transport near the low-order rational tori, thus forming the transport barriers.

\section{FORMATION OF TRANSPORT BARRIERS}

We study the transport barrier formation near the low-order rational tori for the two kinds of profiles of $\omega(J)$ described in Sec. II D: (i) the monotonic dependence of $1 / \omega(J)$ on $J$, and (ii) the nonmonotonic dependence of $1 / \omega(J)$ on $J$ with a minimum at $J=J_{m}$ located away from the center $J=0$ and the edge $J=1$.

\section{A. Monotonic profile}

The profile of $D$ along the variable $J$ is shown in Fig. 7 in the whole region $0<J<1$ for the large Kubo number $K=100$ (a) and in the region $0.5<J<0.55$ located near the rational tori $\omega=1 / 3$ for the several values of $K=1,2,5$, and 100 (b). The values for the other parameters are chosen: $q_{0}=0.8, q_{a}=5, \gamma \equiv 1,20 \leqslant n \leqslant 50$, and $\alpha=5 / 6$, which corresponds to the power spectrum $\left|H_{m n}\right|^{2} \sim n^{-5 / 3}$, and the

\footnotetext{
${ }^{1}$ The existence of gaps in the density of rational tori has been pointed out already in many above-mentioned works [15-25], however, no quantitative estimations have been presented. Moreover, I could not find the results obtained here in existing literature, although one could expect that this problem has been treated already in number theory or in other fields of physics. One expects that these results can also be obtained using the continued fraction expansions of rational numbers or the Euler's totient function [52].
} 


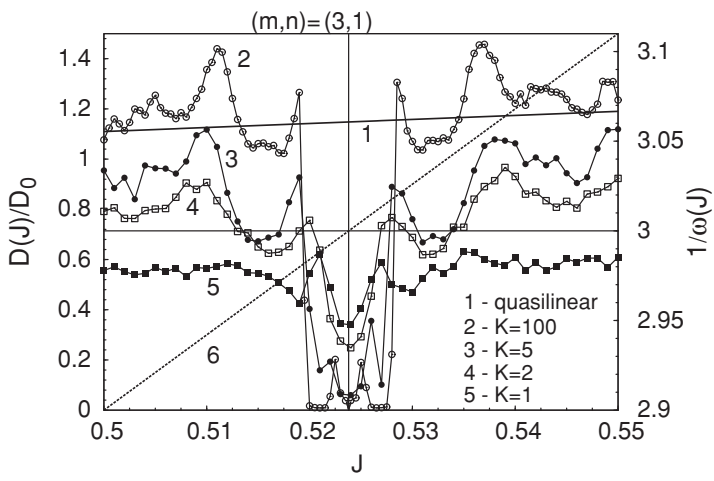

FIG. 8. Expanded view of rectangular area in Fig. 7 near the resonant tori $\omega=1: 3$. Curve 1 (solid) corresponds to the quasilinear prediction, curve 2 (open circles) correspond to the Kubo numbers $K=100$, curves 3 (solid circles), 4 (open squares), and 5 (solid squares) correspond to the Kubo numbers $K=100,5$, 2, and 1, respectively. Dashed curve 6 corresponds to the period of motion $T(J)$ (right-hand axis).

turbulence level $\epsilon=10^{-4}$. At the chosen turbulence level $\epsilon$ the radial transport is mainly caused by the chaotic diffusion.

As seen from Fig. 7 the diffusion coefficient $D$ (curve 2) has a fractal-like dependence which on average follows the quasilinear diffusion coefficient $D(J)$ (21) (curve 1). One can see that the diffusion coefficients drop almost to zero at the radial positions $J=J_{m n}$ corresponding to the low-order rational tori $\omega\left(J_{m n}\right)=n / m$. Near these resonant tori the second moment $\sigma_{0}^{2}(t)$ does not grow with $t$ or grow very slow similar to the one shown in Fig. 2 (curve 1).

It is clear that for the large Kubo numbers $K \gg 1$ the absence of chaotic transport near the low-order rational tori is related to the gaps in the density of rational tori there (see Sec. IV). However, by decreasing the Kubo number the turbulence fluctuations start to cause the transport across low-order rational tori.

The latter is illustrated in Fig. 8 by the expanded view of the profiles of $D(J)$ in the region $0.5 \leqslant J \leqslant 0.55$ located near the rational tori $J=J_{m n},(m, n)=(3,1)$, for the different Kubo numbers $K$. It shows how the transport barrier is changed with the Kubo number $K$. The maximal depth of the barrier is achieved for the large Kubo number $K$ as shown by curve 2 (open circles) for $K=100$. A decrease of the Kubo number $K$ leads to the shrink of the barrier depth without a significant change of its width [see curves 3 (solid circles)], 4 (open squares), and 5 (solid squares) corresponding to the Kubo numbers $K=5,2$, and 1 , respectively. One can expect that the transport barrier disappears for the values of the Kubo number $K<1$. The transport barriers do not disappear with increasing the maximal mode number $n_{\max }$ and decreasing $n_{\min }$ up to 1 . These changes only affect the depth and the width of transport barriers.

The transport barrier also does not disappear by increasing the exponent $\alpha$ in the power-law spectrum $c_{n}=n^{-\alpha}$. However, because of a fast decay of the amplitudes of higher modes $n$ with increasing $\alpha$ the critical turbulence level $\epsilon$ for the chaotic transport also grows. The example of this is shown Fig. 9 for the value $\alpha=2$ and the turbulence level $\epsilon=10^{-2}$. From the comparison with Fig. 7(b) it follows that the magnitude

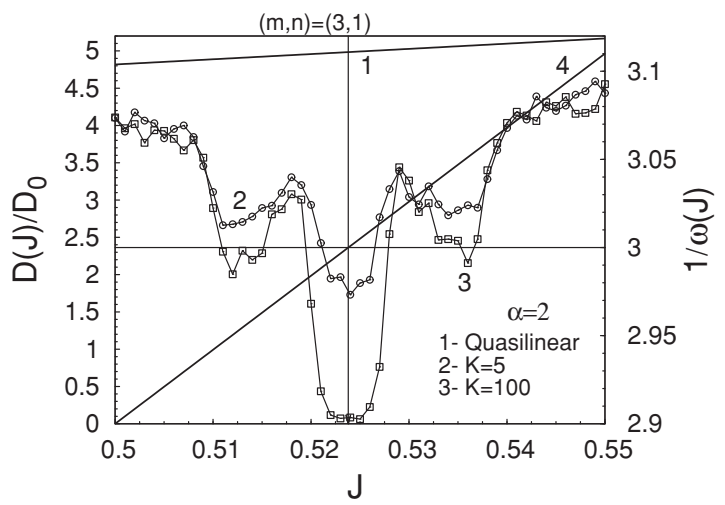

FIG. 9. Profile of the diffusion coefficients near the resonant tori $q=3: 1$ for the exponent $\alpha=2$ and the turbulence level $\epsilon=10^{-2}$. Curve corresponds to the quasilinear value, curves 2 (circles) and 3 (squares), correspond to the Kubo numbers $K=5$ and 100 , respectively (left-hand axis); curve 4 describes $1 / \omega(J)$ (right-hand axis). The averaging is done over $10^{4}$ orbits. The normalization constant $D_{0}=10^{-9}$.

of the diffusion rate for $\alpha=5 / 6$ and $\epsilon=10^{-4}$ is reached at the larger value of $\alpha=2$ with the two-order-higher level of turbulence $\epsilon$.

\section{B. Nonmonotonic profile}

From Eq. (25) it follows that the width of transport barriers also depends on the shear $d \omega(J) / d J$. The most pronounced transport barrier appears in the case of the nonmonotonic profile of $1 / \omega(J)$ near the shearless tori $J=J_{m}$ where $1 / \omega(J)$ reaches its minimal value (i.e., $d \omega / d J=0$ ). According to (25) the density distribution $\rho(J, N)$ near this tori is also low. The formation of the turbulent transport barrier in this case is illustrated in Fig. 10 when the minimal value $J_{m}$ coincides with the $(m=3, n=2)$ resonant tori $\omega\left(J_{m n}\right)=2 / 3$ for the large, $K=100$ (curve 2) and small $K=1$ (curve 3 ) values of the Kubo number. The parameters of the model are $q_{m}=1.5$,

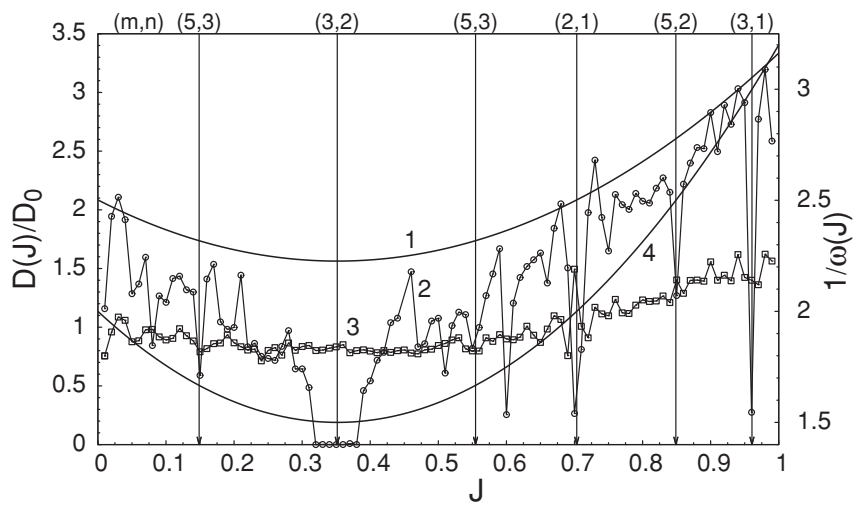

FIG. 10. The same as in Fig. 7 but for the nonmonotonic profile of $1 / \omega(J)=q_{m}+\beta\left(J-J_{m}\right)^{2}$. (a) The turbulence level $\epsilon=10^{-4}$, the interval of modes $10 \leqslant n \leqslant 100$. Curve 1 corresponds to the quasilinear formula (21), curves 2 (circles) and 3 (squares) correspond to the numerical calculations with the Kubo numbers $K=100$ and $K=1$, respectively; curve 4 describes $1 / \omega(J)$ (right-hand axis). The normalization constant $D_{0}=10^{-9}$. 


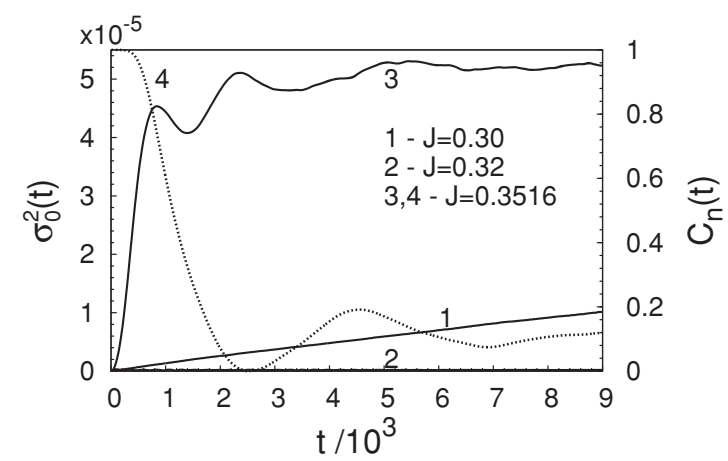

FIG. 11. Dependencies of $\sigma_{0}^{2}(t)$ on $t$ at the three locations of $J$ : curve 1 corresponds to $J_{0}=0.3$; curve $2-J_{0}=0.32$, and curve $3-J_{0}=0.3516$ (left-hand axis); curve 4 (dotted) corresponds to the phase correlation function $C_{n}(t)$ at $n=10$ (right-hand axis). The toroidal modes $10 \leqslant n \leqslant 100$, the turbulence level $\epsilon=10^{-4}$.

$J_{m}=0.3516, \beta=4.045$, the interval of modes $10 \leqslant n \leqslant 100$, the perturbation level is $\epsilon=10^{-4}$, and the values of the other parameters are the same as in Fig. 7.

The typical dependencies of the second moment of displacements $\sigma_{0}^{2}(t)$ on $t$ at the three positions located near this transport barrier is shown in Fig. 11: Curve 1 corresponds to $J=0.3$ located just outside the barrier, curve 2 corresponds to $J=0.32$ located inside the barrier and close to the barrier border, and curve 3 corresponds to the resonance value $J_{m n}=$ 0.3516, $(m=3, n=2)$; curve 4 describes the correlation function of phase $C_{n}(t)$ for the minimal mode number $n=10$. As seen from this figure the motion near the low-order resonant tori is not diffusive, but rather regular. In the transport barrier regions the second moment of displacement, $\sigma_{0}^{2}(J)$, do not linearly depend on time $t$. It grows at the initial stage $t<t_{K}$, and tends to the constant value at the large $t>t_{K}$ (curve 4). The correlation time $t_{K}$ in the transport barrier region is much larger than those in the diffusive regions.

The radial transport barrier in a small-scale turbulent field appears for any turbulence level (but still small) exceeding a certain critical value at which the overlapping of resonances occurs. Only the depth and width of the transport barrier depend on the mode content of the turbulent field. Particularly, the increase of the maximal mode number $n_{\max }$ as well as the increase of the turbulence level $\epsilon$ lead to the growth of diffusion rate at all radial positions due to an increase of the density of rational tori, but it does not eliminate the transport barrier. This is illustrated in Figs. 12 and 13(a). Particularly, Fig. 12 shows the profiles of diffusion coefficients for the three different values of $n_{\max }: n_{\max }=25$ (curve 1 ), $n_{\max }=50$ (curve 2 ), and $n_{\max }=100$ (curve 3 ) at the fixed lowest mode number $n_{\min }=1$ and the Kubo number $K=100$. The turbulence level is taken $\epsilon=10^{-3}$ which is one order higher than in Fig. 10.

From the comparison of Figs. 12 and 10 it is seen that the transport barriers near the shearless tori $(m, n)=(3,2)$ and the resonant tori $(m, n)=(2,1)$ and $(5,2)$ became wider by increasing the turbulence level $\epsilon$ and the inclusion of low-order modes $n_{\min }<10$. At the same time due to a large turbulence level the diffusion inside the barrier does not disappear unlike the case with small $\epsilon$ as in Fig. 10. Widening the transport

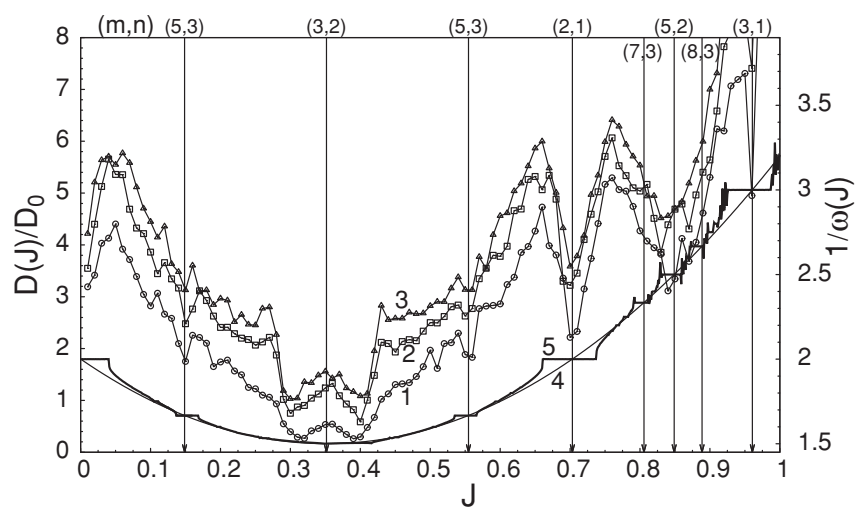

FIG. 12. The same as in Fig. 10 but for the turbulence level $\epsilon=10^{-3}$ : curve 1 (circles) corresponds to the interval of modes $1 \leqslant n \leqslant 25$, curve 2 (squares) to $1 \leqslant n \leqslant 50$, curve 3 (up-triangle) to $1 \leqslant n \leqslant 100$, and curve 4 (dashed) and curve 5 (solid) describe $1 / \omega(J)$ and the inverse of the effective winding number, $1 / \omega_{\text {eff }}(J)$, (27) (right-hand axis). The normalization constant $D_{0}=10^{-7}$ and the Kubo number $K=100$.

barriers near the resonant tori $(m, n)=(2,1)$ and $(5,2)$ are due to the presence of low-order modes $n$. These modes alone modify the winding number $\omega(J)$ near the low-order rational tori because of the trapping of particles into the corresponding resonances. The motion there can be characterized by the effective winding number $\omega_{\text {eff }}(J)$,

$$
\frac{1}{\omega_{\text {eff }}}=\frac{1}{N} \sum_{k=0}^{N} \frac{t_{k+1}-t_{k}}{2 \pi}, \quad N \gg 1,
$$

where $t_{k}(k=0,1,2, \ldots, N)$ is a sequence of times in the mapping (9), and the averaging is done over a large number of mapping iterations $N, N \gg 1$. The profile of $\omega_{\text {eff }}(J)$ is flattened in the region $J_{m n}-\Delta J_{m n} / 2<J<J_{m n}+\Delta J_{m n} / 2$ near the resonant tori $J_{m n}$, where $\Delta J_{m n}$ is the resonance width (20). This is shown by curve 5 in Fig. 12 calculated for the Hamiltonian system (1)-(3) with only the three lowest modes $n=1,2,3$. According to (25), such a profile of $\omega_{\text {eff }}$ leads to widening of the gap in the density of rational tori, and thus reduces the chaotic transport like near the shearless tori.

Figure 13(a) shows the dependence of diffusion coefficients on $n_{\max }$ at the two different radial locations, at the fixed Kubo number $K=100$. As seen from Fig. 13(a) the difference of diffusion coefficients at the two positions located just outside the transport barrier $(J=0.25$, curve 1$)$ and just inside the transport barrier $(J=0.31$, curve 2$)$ remains almost constant with increasing $n_{\max }$ up to 3200 . From Fig. 13 also follows that the diffusion inside the transport barrier appears when $n_{\text {max }}$ exceeds a certain critical number larger than $n_{\max }^{\text {(crit) }} \approx 15$.

The transport barrier near the shearless tori disappears for the small Kubo number $K$. This is shown in Fig. 13(b) where the dependencies of diffusion coefficients on $K$ at the two radial positions located just outside the transport barrier $(J=$ 0.25 , curve 1$)$ and just inside the transport barrier $(J=0.31$, curve 2) are plotted for the turbulent level $\epsilon=10^{-3}$ and the modes $1 \leqslant n \leqslant 50$. As seen from Fig. 13(b) at these conditions 


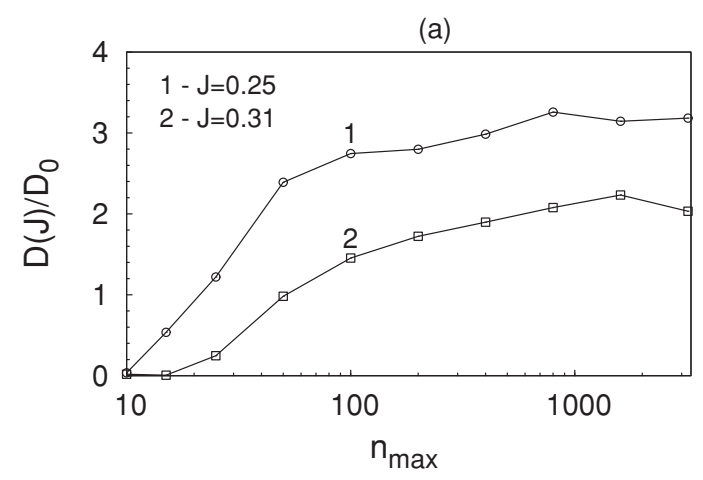

(b)

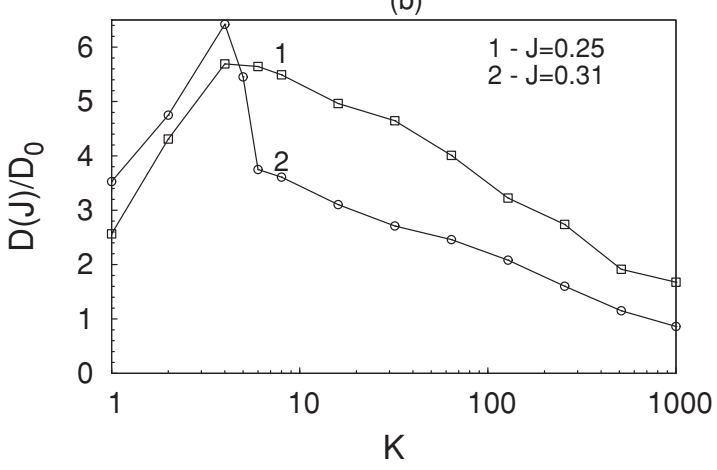

FIG. 13. (a) Dependence of diffusion coefficients on the maximal mode number $n_{\max }$ at the fixed Kubo number $K=100$ at the two radial positions near the transport barrier border shown in Fig. 10(b). Curve 1 corresponds to $J_{0}=0.25$ and curve $2-$ to $J_{0}=0.31$. (b) Dependence of diffusion coefficients on the Kubo number $K$ at the fixed mode interval $1 \leqslant n \leqslant 50$ at the same radial positions as in (a).

the transport barrier appears for the Kubo number $K>6$. For the value $K=1$ the transport barrier near the shearless tori $\omega=2 / 3$ completely disappears (see also curve 3 in Fig. 10).

\section{CONCLUSIONS}

We have studied the chaotic transport in a Hamiltonian system perturbed by a weak turbulent field with a spectrum of modes which contains up to thousands of small-scale wave modes. The numerical calculations of diffusion coefficients are carried out using a fast running mapping technique which overcomes the difficulties of the symplectic integrator with small time steps related to huge computational times. The profiles of diffusion coefficients $D(J)$ across tori $J$ are calculated for the different mode contents and the Kubo numbers. It is found that for the large Kubo numbers the dependence of $D(J)$ on $J$ has an irregular, fractal-like behavior with the reduced and zero diffusivity near the low-order rational tori thereby forming the transport barriers for the chaotic motion. For the small Kubo numbers the irregular behavior of $D(J)$ is replaced by the more smooth profiles, and particularly, the transport barriers may be disappeared or weakened. The dependence of the width and depth of transport barriers on the mode spectra $H_{m n}$, mode ranges in $n$, and the Kubo number $K$ are investigated.

The appearance of transport barriers is related to the gaps of density of rational tori near the low-order rational tori.
The latter is determined by the shear of the unperturbed Hamiltonian system and the density of rational numbers, $m / n$, with $n$ in $n_{\min } \leqslant n \leqslant n_{\max }$, where $n_{\min }$ and $n_{\max }$ are the minimal and maximal mode numbers of the turbulent field. By the numerical calculations of the density of rational numbers we have studied the dependence of the gaps on the maximal mode number. Particularly, the self-similar behavior of the density near the low-order rational numbers has been found.

The transport barriers may not be formed at the large level of turbulent field. As the thorough analytical and numerical analyses of the particle transport in a prescribed broad wave field with random phases made in Ref. [47] (and references therein) shows that the transport has a diffusive nature and the diffusion coefficients take a quasilinear value at small and large turbulence levels.

The fractal behavior of the diffusion coefficients also has been observed in dynamical systems described by onedimensional, piecewise linear, chaotic mappings [48,49]. In these systems the diffusion coefficients have a fractal structure as a function of the mapping parameter.

One should note some differences in the transport barrier formation in the considered Hamiltonian system with the large Kubo number $(K \gg 1)$ from the ones with a small number of perturbation modes, for example, the standard map (see, e.g., [39]). In the former the transport barriers are formed near the low-order rational tori, while in the latter the main obstacle to the global chaotic transport is due to the existence of robust Kolmogorov-Arnold-Moser (KAM) tori corresponding to the most irrational winding numbers $\omega\left(J_{m n}\right)$, with the notable golden KAM tori $g *=(\sqrt{5}-1) / 2$ [50]. On the other hand, in Hamiltonian systems subjected to the nonsmooth perturbations the chaotic transport occurs at any small magnitude of perturbation. The perturbation Hamiltonian of such systems contains an infinite number of modes with the regular phases $\chi_{m n}$ and the amplitudes $H_{m n} \sim|m|^{-\alpha},(\alpha \leqslant 2)[51]$ (see also [40]).

The transport barrier formation near the low-order rational tori found numerically in Hamiltonian systems perturbed by a weak turbulent field at the large Kubo numbers, probably, may have some sort of relation to the transport barriers in fusion experiments (see, e.g., a review [4]). As was mentioned above there is a possible relation between the density of rational magnetic surfaces (or tori) and the transport of particles and energy, particularly, the transport barrier formation in fusion experiments, which has been already discussed in a number of papers [12-25]. Unlike qualitative arguments given in those works we have explicitly shown the formation of transport barriers in Hamiltonian systems by direct numerical calculations and explored the conditions at which such barriers are created. However, our test particle approach is not able to self-consistently tackle this complex nonlinear problem. Therefore, our mechanism cannot be considered as a sole responsible mechanism for the formation of transport barriers in fusion plasmas.

\section{ACKNOWLEDGMENTS}

The author is grateful to D. F. Escande for reading the manuscript and useful suggestions, and M. Z. Tokar for interesting discussions. 
[1] J. Wesson, Tokamaks, 3rd ed. (Clarendon Press, Oxford, 2004).

[2] X. Garbet, C. R. Phys. 7, 573 (2006).

[3] T. Tala and X. Garbet, C. R. Phys. 7, 622 (2006).

[4] E. Doyle et al., Nucl. Fusion 47, S18 (2007).

[5] W. Baumjohann and R. A. Treumann, Basic Space Plasma Physics (Imperial College Press, London, 1997).

[6] J. Jokipi, Adv. Space Res. 23, 617 (1999).

[7] H. Fichtner, Adv. Space Res. 35, 512 (2005).

[8] F. Wagner et al., Phys. Rev. Lett. 49, 1408 (1982).

[9] K. H. Burrel, Plasma Phys. Controlled Fusion 36, A291 (1994).

[10] P. Gohil, C. R. Phys. 7, 606 (2006).

[11] R. Wolf, Plasma Phys. Controlled Fusion 45, R1 (2003).

[12] A. Beklemishev and W. Horton, Phys. Fluids B 4, 200 (1992).

[13] N. J. L. Cardozo et al., Plasma Phys. Controlled Fusion 39, B303 (1997).

[14] G. Hogeweij, N. L. Cardozo, M. D. Baar, and A. Schilham, Nucl. Fusion 38, 1881 (1998).

[15] P. Gohil et al., Nucl. Fusion 43, 708 (2003).

[16] L.-G. Eriksson et al., Phys. Rev. Lett. 88, 145001 (2002).

[17] S. V. Kasilov, D. Reiter, A. M. Runov, W. Kernbichler, and M. F. Heyn, Plasma Phys. Controlled Fusion 44, 985 (2002).

[18] K. A. Razumova et al., Plasma Phys. Controlled Fusion 45, 1247 (2003).

[19] N. L. Cardozo, Trans. Fusion Sci. Tech. 45, 321 (2004).

[20] G. Hogeweij et al., Nucl. Fusion 44, 533 (2004).

[21] E. Joffrin et al., Nucl. Fusion 43, 1167 (2003).

[22] M. E. Austin et al., Phys. Plasmas 13, 082502 (2006).

[23] K. Razumova et al., Plasma Phys. Controlled Fusion 50, 105004 (2008).

[24] R. Brakel and the W7-AS Team, Nucl. Fusion 42, 903 (2002).

[25] X. Garbet et al., Nucl. Fusion 43, 975 (2003).

[26] B. Weyssow, J. H. Misguich, and R. Balescu, Plasma Phys. Controlled Fusion 33, 763 (1991).

[27] Y. Nishimura and M. Azumi, Phys. Plasmas 4, 2365 (1997).

[28] W. Horton et al., Phys. Plasmas 5, 3910 (1998).

[29] M. deRover, N. J. LopesCardozo, and A. Montvai, Phys. Plasmas 3, 4468 (1996).
[30] M. deRover, N. J. LopesCardozo, and A. Montvai, Phys. Plasmas 3, 4478 (1996).

[31] M. de Rover, A. M. Schilham, A. Montvai, and N. J. Lopes Cardozo, Phys. Plasmas 6, 2443 (1999).

[32] J. M. Kwon et al., Phys. Plasmas 7, 1169 (2000).

[33] F. Miskane et al., Eur. Phys. J.: Appl. Phys. 13, 205 (2001).

[34] M. El Mouden, D. Saifaoui, A. Dezairi, B. Zine, and M. Eddahby, J. Plasma Phys. 73, 439 (2007).

[35] M. Vlad and F. Spineanu, in Test Particles, Test Modes and Drift Turbulence, AIP Conf. Ser., Vol. 1061 (AIP, Melville, 2008), pp. 24-33.

[36] G. Michalek, Astron. Astrophys. 376, 667 (2001).

[37] S. Ferreira, Adv. Space Res. 35, 586 (2005),

[38] A. Montvai and D. F. Düchs, in Proceedings Physics Computing'92, Prague, 1992 (World Scientific, Singapore, 1993), p. 417.

[39] A. J. Lichtenberg and M. A. Lieberman, Regular and Stochastic Motion (Springer, New York, 1992).

[40] S. S. Abdullaev, Construction of Mappings for Hamiltonian Systems and their Applications (Springer-Verlag, Berlin/ Heidelberg, 2006).

[41] S. S. Abdullaev, J. Phys. A 32, 2745 (1999).

[42] S. S. Abdullaev, J. Phys. A 35, 2811 (2002).

[43] S. S. Abdullaev, Nucl. Fusion 50, 034001 (2010).

[44] S. S. Abdullaev, Phys. Rev. E 70, 046202 (2004).

[45] S. S. Abdullaev, Phys. Rev. E 72, 046202 (2005).

[46] S. S. Abdullaev, K. Finken, T. Kudyakov, and M. Lehnen, Contrib. Plasma Phys. 50, 929 (2010).

[47] Y. Elskens and D. F. Escande, Microscopic Dynamics of Plasmas and Chaos (Institute of Physics, Bristol, 2003).

[48] R. Klages and J. R. Dorfman, Phys. Rev. Lett. 74, 387 (1995).

[49] G. Knight and R. Klages, Nonlinearity 24, 227 (2011).

[50] J. M. Greene, J. Math. Phys. 20, 1183 (1979).

[51] B. V. Chirikov, Chaos Solitions and Fractals 1, 79 (1991).

[52] M. Abramowitz and I. Stegun, eds., Handbook on Mathematical Functions (Dover Publications, New York, 1965). 